\title{
Transport and magnetic properties of La-doped $\mathrm{CaFe}_{2} \mathrm{As}_{2}$
}

\author{
J. J. Ying, J. C. Liang, X. G. Luo, X. F. Wang, Y. J. Yan, M. \\ Zhang, A. F. Wang, Z. J. Xiang, G. J. Ye, P. Cheng and X. H. Chen* \\ Hefei National Laboratory for Physical Science at Microscale and Department of Physics, \\ University of Science and Technology of China, Hefei, \\ Anhui 230026, People's Republic of China
}

\begin{abstract}
We measured the transport properties and susceptibility of single crystals $\mathrm{Ca}_{1-x} \mathrm{La}_{x} \mathrm{Fe}_{2} \mathrm{As}_{2}$ ( $\mathrm{x}=0$, $0.05,0.1,0.15,0.19$ and 0.25$)$. Large in-plane resistivity anisotropy similar to that in Co-doped 122 iron-pnictides is observed although no transition metals were introduced in the FeAs-plane. The in-plane resistivity anisotropy gradually increases with La doping below $\mathrm{T}_{S D W}$, being different from the hole-doped 122 superconductors. The susceptibilities of the samples show that La doping leads to suppression of SDW and induces a Curie-Weiss-like behavior at low temperature, which is much stronger than the other 122 iron-based superconductors.
\end{abstract}

PACS numbers: 74.25.-q, 74.25.F-, 74.70.Xa

Iron-based superconductors have attracted great attentions these years $1-\underline{\underline{4}}$. The parent compound undergoes structure and spin density wave (SDW) transitions. With chemical doping or high pressure, both structure and SDW transition can be suppressed and superconductivity emerges. $A \mathrm{Fe}_{2} \mathrm{As}_{2}(A=\mathrm{Ca}, \mathrm{Sr}, \mathrm{Ba}, \mathrm{Eu}$, so called "122") with the $\mathrm{ThCr}_{2} \mathrm{Si}_{2}$-type structure were widely investigated because it is easy to grow large size of high quality single crystals. However, the highest Tc of 122 superconductors do not surpass $40 \mathrm{~K}$ in the earlier studies. Recently, superconductivity up to $49 \mathrm{~K}$ was discovered in rare earth doped $\mathrm{CaFe}_{2} \mathrm{As}_{2}{ }^{\underline{5}-8}$. The FeAs plane of $\mathrm{Ca}_{1-x} R_{x} \mathrm{Fe}_{2} \mathrm{As}_{2}$ ( $R=$ rare earth elements) is not affected by substitution of trivalent $R^{3+}$ ions on divalent $\mathrm{Ca}^{2+}$. While for the other two famous electron-doped $122 \mathrm{su}-$ perconductors $\mathrm{BaFe}_{2-x} \mathrm{Co}_{x} \mathrm{As}_{2}$ and $\mathrm{K}_{x} \mathrm{Fe}_{2-y} \mathrm{Se}_{2}$, some of the Fe ions in the FeAs/FeSe layers are either substituted by Co or missed ${ }^{9-11}$. It is an ideal candidate for us to investigate the electron-doped iron-based superconductors with the perfect FeAs layers. The superconducting temperature in $\mathrm{Ca}_{1-x} R_{x} \mathrm{Fe}_{2} \mathrm{As}_{2}$ is much higher compared to the other 122 iron pnictides and it is very necessary for us to detailedly investigate the physical properties of $\mathrm{Ca}_{1-x} R_{x} \mathrm{Fe}_{2} \mathrm{As}_{2}$.

Recent works showed large in-plane resistivity anisotropy below $T_{S}$ or $T_{N}$ in the parent and electrondoped 122 system although the distortion of the orthorhombic structure is less than $1 \% 12-15$ in the SDW state. However, for the hole-doped $\mathrm{Ba}_{1-x} \mathrm{~K}_{x} \mathrm{Fe}_{2} \mathrm{As}_{2}$, inplane resistivity anisotropy is nearly absent $\underline{14}$. All the previous works were focusing on the $\mathrm{Co}, \mathrm{Ni}$ and $\mathrm{Cu}$ substitution on the Fe site for electron-doped samples ${ }^{16}$ and it is supposed that the large in-plane anisotropy might come from the transition metal substitution in the FeAs plane. It is very meaningful for us to study the in-plane resistivity anisotropy for the electron-doped

*Corresponding author; Electronic address: chenxh@ustc.edu.cn
$\mathrm{Ca}_{1-x} R_{x} \mathrm{Fe}_{2} \mathrm{As}_{2}$ with the perfect Fe square lattice. The large in-plane anisotropy observed in $\mathrm{Ca}_{1-x} R_{x} \mathrm{Fe}_{2} \mathrm{As}_{2}$ in this paper indicates that the large in-plane resistivity anisotropy is related to the electron-doping rather than transition metal doped in the FeAs plane.

In this paper, we systematically investigated the transport and magnetic properties of La-doped $\mathrm{CaFe}_{2} \mathrm{As}_{2}$. Superconductivity up to $43 \mathrm{~K}$ was observed in the $\mathrm{Ca}_{0.81} \mathrm{La}_{0.19} \mathrm{Fe}_{2} \mathrm{As}_{2}$ similar to the previous results ${ }^{6}$. SDW transition can be suppressed through La doping and superconductivity coexists with antiferromagnetic with the La doping level between 0.05 and 0.15 . The in-plane resistivity anisotropy gradually increases with La doping below $\mathrm{T}_{S D W}$, being different from the holedoped 122 superconductors. A strong Curie-Weiss-like behavior at low temperature is induced by La-doping.

High quality single crystals with nominal composition $\mathrm{Ca}_{1-x} \mathrm{La}_{x} \mathrm{Fe}_{2} \mathrm{As}_{2}$ (x=0, 0.05, 0.1, 0.15, 0.2 and 0.3) were grown by conventional solid-state reaction using FeAs as self-flux ${ }^{6-8}$. The FeAs precursor was first synthesized from stoichiometric amounts of $\mathrm{Fe}$ and As inside the silica tube at $800{ }^{\circ} \mathrm{C}$ for $24 \mathrm{~h}$. Appropriate amounts of the starting materials of FeAs, Ca and La were placed in an alumina crucible, and sealed in an quartz tube. The mixture was heated to $1180{ }^{\circ} \mathrm{C}$ in 6 hours and then kept at this temperature for 10 hours, and later slowly cooled down to $950{ }^{\circ} \mathrm{C}$ at a rate of $3{ }^{\circ} \mathrm{C} /$ hour. After that, the temperature was cooled down to room temperature by shutting down the furnace. The shining platelike $\mathrm{Ca}_{1-x} \mathrm{La}_{x} \mathrm{Fe}_{2} \mathrm{As}_{2}$ crystals were mechanically cleaved from the flux and obtained for measurements. The actual composition of the single crystals were characterized by the Energy-dispersive X-ray spectroscopy (EDX). The actually doping levels are almost the same with the nominal values for $\mathrm{x}$ smaller than 0.2 . While for the nominal composition $\mathrm{x}=0.2$ and 0.3 , the actual values of $\mathrm{x}$ are 0.19 and 0.25 which are smaller than the nominal values. Resistivity was measured using the Quantum Design PPMS-9 and Magnetic susceptibility was measured using the Quantum Design SQUID-MPMS. In-plane resistivity 

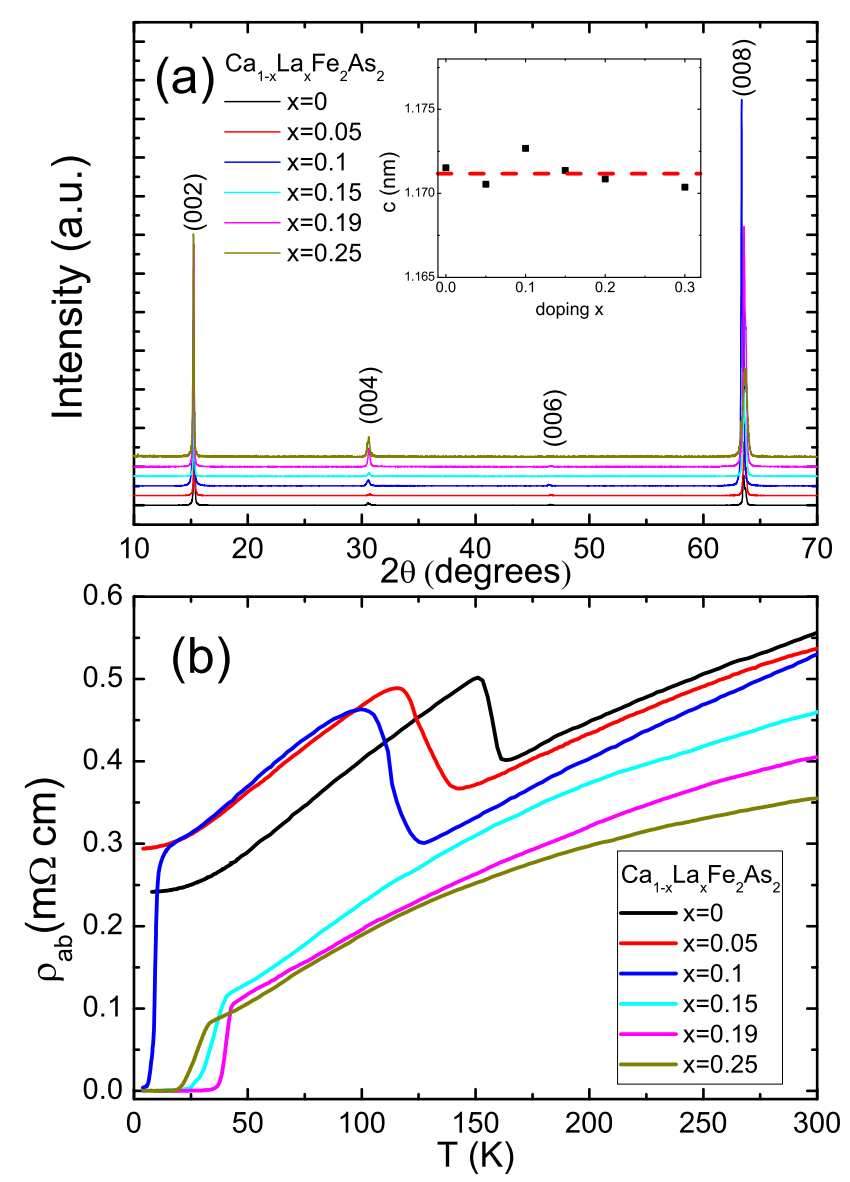

FIG. 1: (Color online)(a): The single crystal x-ray diffraction pattern of $\mathrm{Ca}_{1-x} \mathrm{La}_{x} \mathrm{Fe}_{2} \mathrm{As}_{2}$, Only (00l) diffraction peaks show up, indicating that the $\mathrm{c}$ axis is perpendicular to the plane of the plate. The inset shows that the c parameter does not changes with doping. (b): Temperature dependence of the in-plane resistivity.

anisotropy was measured using the same method with the previous work ${ }^{12,14}$. Crystals were cut parallel to the orthorhombic $\mathrm{a}$ and $\mathrm{b}$ axes so that the orthorhombic $\mathrm{a}(\mathrm{b})$ direction is perpendicular (parallel) to the applied pressure direction. $\rho_{a}$ (current parallel to a) and $\rho_{b}$ (current parallel to b) were measured on the same sample using standard 4-point configuration.

Single crystals of $\mathrm{Ca}_{1-x} \mathrm{La}_{x} \mathrm{Fe}_{2} \mathrm{As}_{2}$ were characterized by x-ray diffractions (XRD) using $\mathrm{Cu} K_{\alpha}$ radiations. As shown in the Fig.1(a). Only (00l) diffraction peaks were observed, suggesting that the crystallographic c axis is perpendicular to the plane of the single crystal. The inset of Fig.1(a) shows the c-axis parameters with different doping level, we can see that the lattice parameters of c-axis almost do not change with La doping. The lattice constant of c-axis was around $11.72 \AA$ for all the samples. It is slightly different from the polycrystalline samples reported previously for which the c-axis slightly decrease with rare earth elements doping ${ }^{5}$. Fig.1(b) shows the
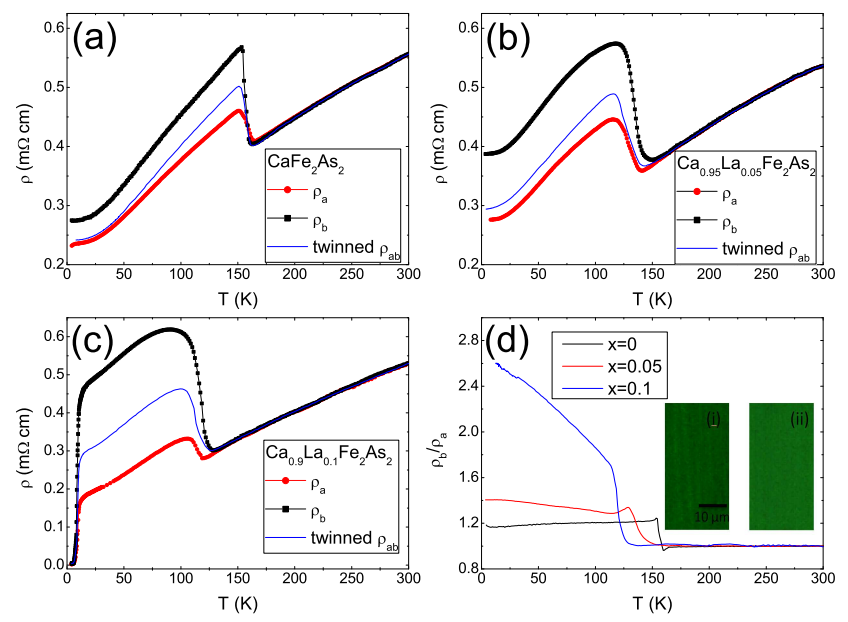

FIG. 2: (Color online) Temperature dependence of in-plane resistivity with the electric current flow along $a$ direction and $b$ direction respectively for (a): parent compound $\mathrm{CaFe}_{2} \mathrm{As}_{2}$, (b): $\mathrm{Ca}_{0.95} \mathrm{La}_{0.05} \mathrm{Fe}_{2} \mathrm{As}_{2}$, (c): $\mathrm{Ca}_{0.9} \mathrm{La}_{0.1} \mathrm{Fe}_{2} \mathrm{As}_{2}$. The twinned in-plane resistivity was also shown for comparison (blue line). (d): Temperature dependence of in-plane resistivity anisotropy $\rho_{b} / \rho_{a}$. The insets of (f) are the Polarized-light images of the surface for twinned(i) and detwinned(ii) $\mathrm{CaFe}_{2} \mathrm{As}_{2}$ at the temperature of $78 \mathrm{~K}$.

temperature dependence of the resistivity with the electric current flowing in the ab plane for $\mathrm{Ca}_{1-x} \mathrm{La}_{x} \mathrm{Fe}_{2} \mathrm{As}_{2}$ single crystals. The upturn of resistivity for parent compound was ascribed to the SDW or structure transition. With La doping, the anomaly was gradually suppressed and superconductivity gradually emerged. For the optimal doped sample $\mathrm{x}=0.19$, the onset of superconductivity was up to $43 \mathrm{~K}$ which is almost the same with the earlier results $\mathrm{f}^{6}$. However, the superconductivity transition in this system is much broader than that in other 122 iron-pnictide superconductors.

Fig.2(a) shows the temperature dependence of in-plane resistivity with the current flowing parallel to the orthorhombic $b$ direction (black) and orthorhombic $a$ direction (red) of the detwinned $\mathrm{CaFe}_{2} \mathrm{As}_{2}$ sample. The insets of Fig.2(d) are the Polarized-light images of the surface for twinned(i) and detwinned(ii) $\mathrm{CaFe}_{2} \mathrm{As}_{2}$ at the temperature of $78 \mathrm{~K}$. The samples are almost fully detwinned as we can no longer see the twin domains. Obvious anisotropy was observed which is similar to the earlier result 13 , although different methods were used to detwin the samples. Compounds with a small amount of La-doping show much larger in-plane anisotropy as displayed in Fig.2(b) and (c) for $\mathrm{x}=0.05$ and $\mathrm{x}=0.1$, respectively. The different behavior of $\rho_{a}$ and $\rho_{b}$ observed here is very similar to the ones in other parent or electronunderdoped iron-pnictides ${ }^{12,13}$. We characterized the degree of in-plane resistivity anisotropy by the ratio $\rho_{b} / \rho_{a}$. Fig.2 (d) shows the temperature dependence of $\rho_{b} / \rho_{a}$ for different doping level samples. For all the samples, inplane resistivity anisotropy increases very quickly below 

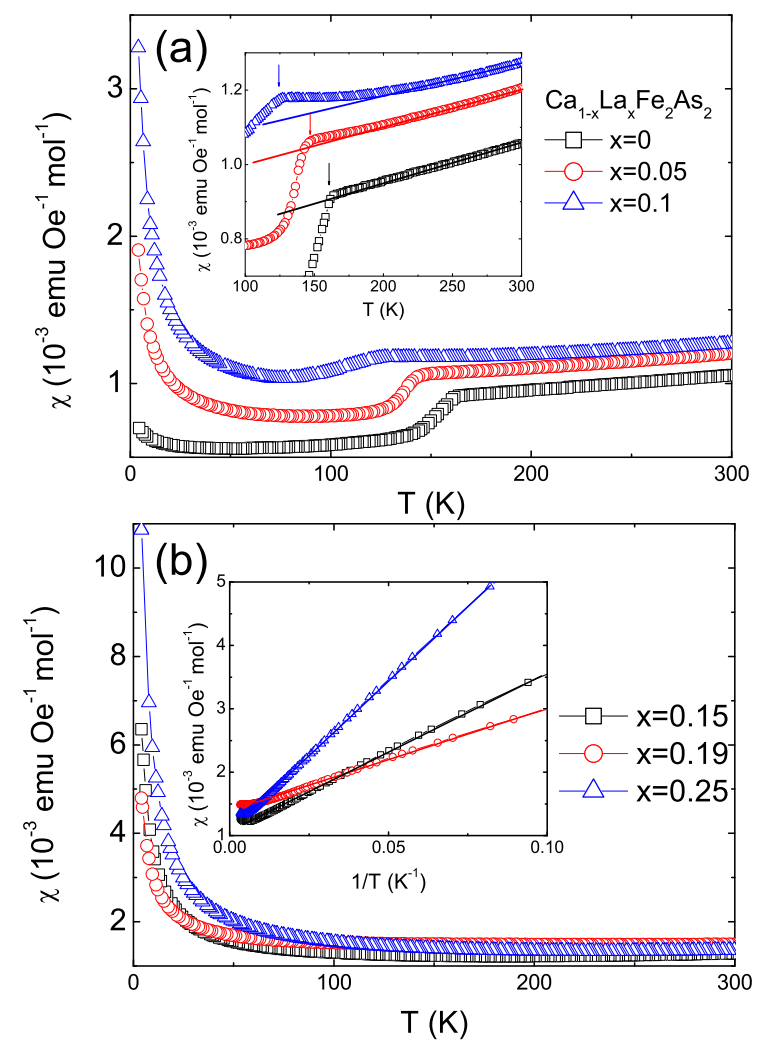

FIG. 3: (Color online) (a): Temperature dependence of magnetic susceptibility for $\mathrm{x}=0,0.05$ and 0.1 . The inset is the enlarged area around $T_{S D W}$. The arrows indicate the $T_{S D W}$. (b): Temperature dependence of magnetic susceptibility for $\mathrm{x}=0.15,0.19$ and 0.25 . The inset shows that the magnetic susceptibility is linear with $1 / \mathrm{T}$ at low temperature, indicating a nice Curiew-Weiss behavior in the low temperature.

$\mathrm{T}_{S D W}$. With increasing La doping level, the in-plane resistivity anisotropy gradually increases in the SDW region. It is very similar with the other underdoped electron-doping 122 systems, but quite different from the hole-doped $\mathrm{Ba}_{1-x} \mathrm{~K}_{x} \mathrm{Fe}_{2} \mathrm{As}_{2}{ }^{14}$. Although the doping position is away from the FeAs plane, large in-plane anisotropy still exists and increases with doping content. The magnitude of in-plane resistivity anisotropy is almost the same as the other underdoped electron-doping 122 samples. These results suggest that the in-plane anisotropy is closely related to the carrier-type rather than the different doping positions.

The temperature dependence of magnetic susceptibilities for $\mathrm{Ca}_{1-x} \mathrm{La}_{x} \mathrm{Fe}_{2} \mathrm{As}_{2}$ ( $\mathrm{x}=0,0.05$ and 0.1 ) with magnetic field of $\mathrm{H}=1 \mathrm{~T}$ applied along the ab-plane are shown in Fig.3 (a). The susceptibility of parent compound $\mathrm{CaFe}_{2} \mathrm{As}_{2}$ shows T-linear behavior and gradually decreases with decreasing temperature above $\mathrm{T}_{S D W}$. The susceptibility suddenly drops at $\mathrm{T}_{S D W}$ and shows very weak Curie-Weiss-like behavior at low temperature.
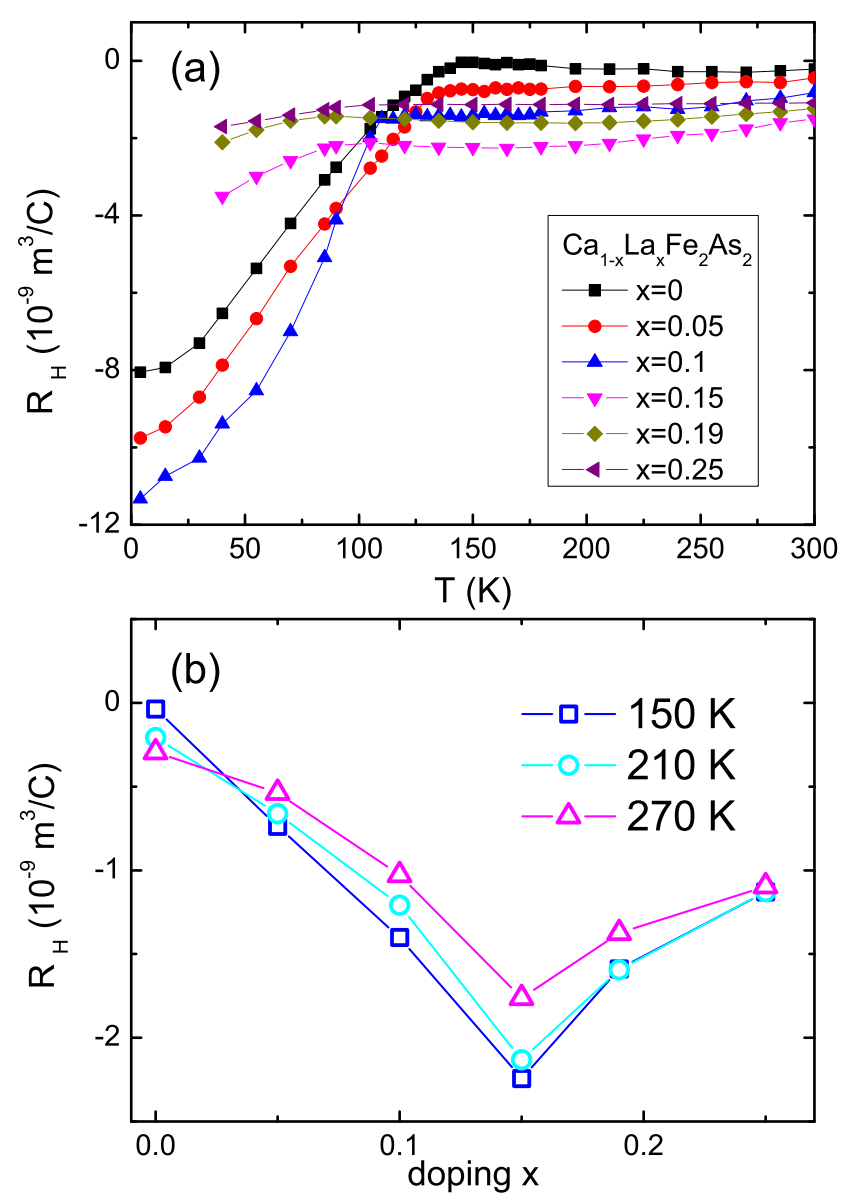

FIG. 4: (Color online) (a): Temperature dependence of $\mathrm{R}_{H}$ for $\mathrm{Ca}_{1-x} \mathrm{La}_{x} \mathrm{Fe}_{2} \mathrm{As}_{2}$. (b): Doping dependence of $\mathrm{R}_{H}$ at certain temperature in the normal state.

This behavior is the same with the previous result 18 . Small amount doping of La obviously enhances the CurieWeiss-like behavior at low temperature. With increasing the doping content, the anomaly due to SDW order is gradually suppressed and T-linear behavior of susceptibility is gradually broken due to the Curie-Weiss-like behavior at low temperature. It strongly contrast to Codoped 122 samples in which the T-linear behavior of susceptibility survives with Co doping17. With further increasing the doping content, the susceptibilities showed strong Curie-Weiss-like behavior below about $200 \mathrm{~K}$ as shown in Fig.3 (b). Such strong Curie-Weiss-like behavior observed in $\mathrm{Ca}_{1-x} \mathrm{La}_{x} \mathrm{Fe}_{2} \mathrm{As}_{2}$ is strongly different to the other 122 iron-pnictide superconductors.

Fig.4(a) shows the temperature dependence of Hall coefficient $\mathrm{R}_{H}$ of $\mathrm{Ca}_{1-x} \mathrm{La}_{x} \mathrm{Fe}_{2} \mathrm{As}_{2}$. $\mathrm{R}_{H}$ of all the samples show the negative values, indicating that the dominated carrier is electron. The sharp increase of the absolute value of $\mathrm{R}_{H}$ below $T_{S D W}$ indicates the sudden drop of carrier density in the SDW state, which is the common 
feature in iron pnictide superconductors ${ }^{19}$. It is also instructive to analyze the Hall Coefficient at certain temperature for different doping level samples at high temperature. It is found that the dependence of doping is nonmonotonic as shown in Fig.4(b). For the samples $\mathrm{x}<0.15$, the absolute value of $\mathrm{R}_{H}$ increases with increasing the doping content. With further doping, it gradually decreases. This behavior is quite similar to the $\mathrm{BaFe}_{2-x} \mathrm{Co}_{x} \mathrm{As}_{2}$ system. Such behavior is caused by the multiband effect and different mobility of electron or hole carriers $^{19}$.

Large in-plane anisotropy is observed in the underdoped region of $\mathrm{Ca}_{1-x} \mathrm{La}_{x} \mathrm{Fe}_{2} \mathrm{As}_{2}$ although no extra elements were introduced into the FeAs-plane. It strongly contrasts to the hole-doped $\mathrm{Ba}_{1-x} \mathrm{~K}_{x} \mathrm{Fe}_{2} \mathrm{As}_{2}$ system, but is similar to the transition metal substitution samples ${ }^{12,14}$. Our results show that substitution away from the FeAs plane could also lead to large in-plane anisotropy, which indicate that the in-plane anisotropy is strongly dependent on doping carriers rather than the doping site. The in-plane anisotropy was suggested closely related to the the orbital degree of freedom ${ }^{20,21}$ or/and spin fluctuations 22 . Our previous work demonstrates that the microscopic orbital involvement in magnetically ordered state must be fundamentally different between the hole and electron underdoped iron pnictides 14 . Large in-plane anisotropy observed in rare earth doped materials also prove this. ARPES experiments need to do to compare the orbital characters of the hole and electron doped samples. The magnetic susceptibilities of $\mathrm{Ca}_{1-x} \mathrm{La}_{x} \mathrm{Fe}_{2} \mathrm{As}_{2}$ show much stronger CurieWeiss-like behavior compared to the other 122 iron pnictide superconductors. Although superconductivity above $40 \mathrm{~K}$ was observed in this system which is much higher than the other 122 superconductors, superconducting transition is broad. The origins of the strong CurieWeiss-like behavior and broad superconducting transition in this system are still unknown.

In conclusion, we systematically measured the transport properties and susceptibilities of $\mathrm{Ca}_{1-x} \mathrm{La}_{x} \mathrm{Fe}_{2} \mathrm{As}_{2}(\mathrm{x}=0, \quad 0.05, \quad 0.1, \quad 0.15, \quad 0.19$ and $0.25)$. Large in-plane anisotropy is observed which is similar to Co-doped 122 iron-pnictides, but strongly contrasts to the hole-doped $\mathrm{Ba}_{1-x} \mathrm{~K}_{x} \mathrm{Fe}_{2} \mathrm{As}_{2}$. Strong Curie-Weiss-like behavior of susceptibility was observed at low temperature by increasing the La doping.

ACKNOWLEDGEMENT This work is supported by the National Basic Research Program of China (973 Program, Grant No. 2012CB922002 and No. 2011CB00101), National Natural Science Foundation of China (Grant No. 11190020 and No. 51021091), the Ministry of Science and Technology of China, and Chinese Academy of Sciences.
1 Y. Kamihara, T. Watanabe, M. Hirano, and H. Hosono, J. Am. Chem. Soc. 130, 3296(2008).

2 X. H. Chen, T. Wu, G. Wu, R. H. Liu, H. Chen and D. F. Fang, Nature 453, 761(2008).

3 Z. A. Ren, G. C. Che, X. L. Dong, J. Yang, W. Lu, W. Yi, X. L. Shen, Z. C. Li, L. L. Sun, F. Zhou and Z. X. Zhao, Europhys. Lett. 83, 17002(2008).

${ }^{4}$ M. Rotter, M. Tegel, D. Johrendt, Phys. Rev. Lett. 101, 107006(2008).

5 S. R. Saha, N. P. Butch, T. Drye, J. Magill, S. Ziemak, K. Kirshenbaum, P. Y. Zavalij, J. W. Lynn, and J. Paglione, arXiv:1105.4798

6 Zhaoshun Gao, Yanpeng Qi, Lei Wang, Dongliang Wang, Xianping Zhang, Chao Yao, Chunlei Wang, Yanwei Ma, EPL, 95, 67002 (2011).

7 B. Lv, L. Z. Deng, M. Gooch, F. Y. Wei, Y. Y. Sun, J. Meen, Y. Y. Xue, B. Lorenz and C. W. Chu, Proc. Nat. Acad. Sci. 108, 15705 (2011).

8 Yanpeng Qi, Zhaoshun Gao, Lei Wang, Dongliang Wang, Xianping Zhang, Chao Yao, Chunlei Wang, Chengduo Wang, Yanwei Ma, arXiv:1106.4208

9 Athena S. Sefat, Rongying Jin, Michael A. McGuire, Brian C. Sales, David J. Singh, and David Mandrus, Phys. Rev. Lett. 101, 117004 (2008).

10 J. Guo, S. Jin, G. Wang, S. Wang, K. Zhu, T. Zhou, M. He and X. Chen, Phys. Rev. B 82, 180520(R) (2010).

11 J. J. Ying, X. F. Wang, X. G. Luo, A. F. Wang, M. Zhang, Y. J. Yan, Z. J. Xiang, R. H. Liu, P. Cheng, G. J. Ye, X. H. Chen , Phys. Rev. B 83, 212502 (2011).

12 Jiun-Haw Chu, James G. Analytis, Kristiaan De Greve,
Peter L. McMahon, Zahirul Islam, Yoshihisa Yamamoto, Ian R. Fisher, Science 329, 824 (2010).

13 M. A. Tanatar, E. C. Blomberg, A. Kreyssig, M. G. Kim, N. Ni, A. Thaler, S. L. Budko, P. C. Canfield, A. I. Goldman, I. I. Mazin, and R. Prozorov, Phys. Rev. B 81, 184508 (2010).

14 J. J. Ying, X. F. Wang, T. Wu, Z. J. Xiang, R.H. Liu, Y. J. Yan, A. F. Wang, M. Zhang, G. J. Ye, P. Cheng, J. P. $\mathrm{Hu}$, and X.H. Chen, Phys. Rev. Lett. 107, 067001 (2011).

15 I R Fisher, L Degiorgi and Z X Shen, Rep. Prog. Phys. 74, 124506 (2011).

16 Hsueh-Hui Kuo, Jiun-Haw Chu, Scott C. Riggs, Leo Yu, Peter L. McMahon, Kristiaan De Greve, Yoshihisa Yamamoto, James G. Analytis, and Ian R. Fisher, Phys. Rev. B 84, 054540 (2011).

17 X. F. Wang, T. Wu, G. Wu, R. H. Liu, H. Chen, Y. L. Xie, X. H. Chen, New J. Phys. 11, 045003 (2009).

18 G Wu, H Chen, TWu, Y L Xie, Y J Yan, R H Liu, X FWang, J J Ying and X H Chen, J. Phys.: Condens. Matter 20422201 (2008)

19 Lei Fang, Huiqian Luo, Peng Cheng, Zhaosheng Wang, Ying Jia, Gang Mu, Bing Shen, I. I. Mazin, Lei Shan, Cong Ren, and Hai-Hu Wen, Phy. Rev. B 80, 140508(R) (2009).

20 C.-C. Chen, J. Maciejko, A. P. Sorini, B. Moritz, R. R. P. Singh, and T. P. Devereaux, Phys. Rev. B 82, 100504(R) (2010).

21 Weicheng Lv, Frank Krger, and Philip Phillips, Phys. Rev. B 82, 045125 (2010)

22 R. M. Fernandes, L. H. VanBebber, S. Bhattacharya, P. 
Chandra, V. Keppens, D. Mandrus, M. A. McGuire, B. C. Sales, A. S. Sefat, and J. Schmalian, Phys. Rev. Lett. 105,

157003 (2011). 